\title{
TIV Contrast Source Inversion of mCSEM data
}

\author{
Torgeir Wiik*, Lars Ole Løseth ${ }^{\dagger}$, Bjørn Ursin* and Ketil Hokstad ${ }^{\dagger}$ \\ * Norwegian University of Science and Technology, \\ Department for Petroleum Engineering and Applied Geophysics, \\ S.P. Andersens vei $15 A$, \\ NO-7491 Trondheim, Norway \\ torgeir.wiik@ntnu.no \\ bjorn.ursin@ntnu.no \\ $\dagger$ Statoil Research Center, \\ Arkitelt Ebbells veg 10, \\ NO-7053 Ranheim, Norway \\ lalo@statoil.com \\ kehok@statoil.com
}

(March 2, 2010)

Running head: TIV Contrast Source Inversion

\begin{abstract}
We present a Contrast Source Inversion (CSI) scheme for electromagnetic data in conductive media. We consider only contrasts in electric conductivity, but allow the
\end{abstract}


medium to be transversely isotropic in the vertical direction (TIV). This has applications for instance in inversion of marine Controlled Source Electromagnetic data. The CSI method is based on the integral equation formulation of electromagnetic field propagation, and we show how the method solves the inverse problem of determining the conductivity structure of the subsurface. The method minimizes a cost functional which enforces data fidelity and that the solution should satisfy the Lippmann-Schwinger equation. Further regularization is introduced linearly into the cost functional to incorporate prior model information. Although the problem is non-linear we choose a strategy of splitting the minimization problem into two linear problems, which are solved alternatingly. To this end contrast sources are introduced, which may be interpreted as sources emitting the scattered field from a scattering object. This approach should yield a computationally fast method compared to solving the non-linear system of equations. Two real field examples are inverted, which demonstrate the method and how the TIV inversion performs compared to isotropic inversion. We find that the CSI method is applicable to real field examples, and the results show that a TIV inversion is preferred over isotropic in order to identify weak anomalies in these examples. The reason for this is that both the horizontal and vertical conductivity affects the signal propagation in the overburden. 


\section{INTRODUCTION}

The use of marine Controlled Source Electromagnetic (mCSEM) data in hydrocarbon prospecting is based on the assumption that hydrocarbon saturated formations possess a significantly lower electric conductivity compared to its surroundings. The EM field emitted from a controlled source travelling inside this resistive region will be less attenuated at far source/receiver offsets than the field propagating in water saturated formations, and the effect of such resistive areas should thus be recognizable at far offsets (Eidesmo et al., 2002). The mCSEM experiment is usually performed by towing a horizontal electric dipole behind a vessel. The dipole typically outputs a square wave current which is the source of electromagnetic signals in the frequency range $0.1 \mathrm{~Hz}-10 \mathrm{~Hz}$. The emitted field travels in the subsurface, before it is recorded when it reaches the seabed (Eidesmo et al., 2002). See Figure 1 for a graphical description, along with the main signal pathways. However, interpretation of mCSEM is not necessarily straightforward. In order to explain the recorded data, imaging algorithms that solves the inverse scattering problem of determining the subsurface parameters are helpful.

EM inverse scattering is a classical topic and has been investigated earlier, see for instance Colton and Kress (1992). In particular, solving both the forward and inverse scattering problem has attracted increased interest from the geophysical society in the later years, treating both crosswell and mCSEM experiments. See for instance Ursin (1983); Wannamaker et al. (1984); Ward and Hohmann (1987); Kong et al. (2008);

Abubakar et al. (2008a); Løseth and Ursin (2007) for modeling, and Newman (1995); 
Newman and Hoversten (2000); Zhdanov (2002); Abubakar et al. (2008a) and the references therein, for inversion. However, mostly isotropic inversion is considered.

We wish to apply the so called Contrast Source Inversion (CSI) method, extended to media which are transversely isotropic in the vertical direction (TIV), in the $\mathrm{mC}$ SEM setting. This means considering media in which the horizontal and vertical conductivities may differ. Anisotropic effects are present in recorded data and hence the isotropic assumption may not be sufficient. The isotropic method has been investigated in several other settings earlier, see for instance van den Berg and Kleinman (1997); Abubakar and van den Berg (2002); Abubakar et al. (2004a). We use the integral equation framework described earlier by e.g. Wannamaker et al. (1984); Colton and Kress (1992); Zhdanov (2002); Abubakar and van den Berg (2004), but the method has also been explored using finite differences (Abubakar et al., 2008b). The method introduces so called contrast sources, which may be interpreted as sources emitting signals that is really scattered from a scattering object. Based on these contrast sources we alternatingly minimize a cost functional with respect to the contrast sources and the contrast between the real medium and a chosen background medium, respectively. The final goal is to obtain an estimate of the contrast which describes the difference between the chosen background and the estimated medium. Thus, the contrast sources are only needed as intermediate values.

Solving the electromagnetic inverse problem is a notoriously ill-posed problem (Feng et al., 2003; Abubakar et al., 2004b) in the Hadamard sense (McOwen, 1996). By this we mean that that the problem might not depend continuously on the initial 
conditions and that there exists several solutions. Hence, there is a need for regularization to enforce a physically meaningful solution. Such regularization may be introduced in several ways, for instance multiplicatively into the cost functional, as described by Abubakar and van den Berg (2004), or linearly (Tikhonov and Arsenin, 1977). We will here introduce a regularizer linearly into the cost functional, which enforces the contrast estimate to remain close to a reference model in some sense, described by weights. We use weights corresponding to a second order smoothing filter and a zero reference model, thus emphasizing the smoothness of the reconstruction, in order to stabilize the numerical calculations. The weights may also be specified, as described later, to incorporate prior model information.

We apply the CSI method to two real field examples. The results show the importance of including anisotropy in the inversion and of considering the information content in the recorded data and the regularizer's nature before performing an inversion. We find that the CSI method is applicable to real field examples, and in these examples a TIV inversion is preferred over isotropic in order to identify weak anomalies. This is because both the horizontal and vertical conductivities affect the field propagation in the overburden (Løseth, 2007). 


\section{THEORY}

\section{Forward scattering}

Consider a background medium described by its electromagnetic parameters

$$
\sigma_{\mathbf{0}}=\left(\begin{array}{ccc}
\sigma_{0, h} & 0 & 0 \\
0 & \sigma_{0, h} & 0 \\
0 & 0 & \sigma_{0, v}
\end{array}\right)=\sigma_{0, v}\left(\begin{array}{ccc}
\Upsilon & 0 & 0 \\
0 & \Upsilon & 0 \\
0 & 0 & 1
\end{array}\right)
$$

$\epsilon_{0}=\epsilon_{0} \mathbf{I}$ and $\mu_{0}=\mu_{0} \mathbf{I}$, where $\mathbf{I}$ is the identity tensor. Here $\sigma_{0, h}$ and $\sigma_{0, v}$ denote the vertical and horizontal conductivities, which may differ in a TIV medium. These parameters are assumed to have some spatial dependence. We accept Ohm's law, which states (Stratton, 1941)

$$
\mathbf{j}=\sigma \mathbf{e}
$$

where $\mathbf{e}$ is the electric field. The electric and magnetic field satisfies Maxwell's equations, which in the frequency domain then read (Stratton, 1941)

$$
\begin{aligned}
\nabla \times \mathbf{e} & =\mathrm{i} \omega \mu_{0} \mathbf{h}, \\
\nabla \times \mathbf{h} & =\tilde{\sigma_{0}} \mathbf{e}+\mathbf{j}^{\mathrm{s}} .
\end{aligned}
$$

where $\tilde{\sigma_{0}}=\sigma_{0}-\mathrm{i} \omega \epsilon_{0} \mathbf{I}$ and $\mathbf{j}^{\mathrm{s}}$ is the electric source current. Here $\mathrm{i}=\sqrt{-1}$ and $\omega$ denotes the angular frequency.

Green's tensors generalizes the concept of Green's functions for scalar equations to vector valued equations (Green, 1828; McOwen, 1996). Physically they represent 
fields from a point source, and the Green's tensors in 3 dimensions are thus represented by $3 \times 3$-matrices with element $(i, j)$ representing the field in $i$-direction from a point source polarized in the $j$-direction, $i, j \in\{x, y, z\}$ (or some other basis for $\mathbb{R}^{3}$ ). This implies that the electric and magnetic Green's tensors due to an electric point source are solutions of the equations

$$
\begin{aligned}
\nabla \times \mathbf{G}^{E}\left(\mathbf{x}, \mathbf{x}_{\mathbf{0}}\right) & =\mathrm{i} \omega \mu_{0} \mathbf{G}^{H}\left(\mathbf{x}, \mathbf{x}_{\mathbf{0}}\right) \\
\nabla \times \mathbf{G}^{H}\left(\mathbf{x}, \mathbf{x}_{\mathbf{0}}\right) & ={\tilde{\sigma_{0}}}^{E}\left(\mathbf{x}, \mathbf{x}_{\mathbf{0}}\right)+\mathbf{I} \delta\left(\mathbf{x}-\mathbf{x}_{\mathbf{0}}\right),
\end{aligned}
$$

where $\mathbf{G}^{E}$ and $\mathbf{G}^{H}$ are the electric and magnetic Green's tensors, respectively, $\nabla \times$ is the dyadic operator corresponding to the well-known vector-curl operator, $\delta$ is Dirac's delta function and $\mathbf{x}_{\mathbf{0}}$ is the source position.

The solution to equations 3 and 4 for some external, electric source with currentsignature $\mathbf{j}^{\mathrm{s}}$ is then given component wise by (Zhdanov, 2002)

$$
\begin{aligned}
\mathbf{e}_{i}(\mathbf{x}) & =\int_{\mathbb{R}^{3}} \mathbf{G}_{i j}^{E}\left(\mathbf{x}, \mathbf{x}^{\prime}\right) \mathbf{j}_{j}^{\mathrm{s}}\left(\mathbf{x}^{\prime}\right) \mathrm{d} \mathbf{x}^{\prime}, \\
\mathbf{h}_{i}(\mathbf{x}) & =\int_{\mathbb{R}^{3}} \mathbf{G}_{i j}^{H}\left(\mathbf{x}, \mathbf{x}^{\prime}\right) \mathbf{j}_{j}^{\mathrm{s}}\left(\mathbf{x}^{\prime}\right) \mathrm{d} \mathbf{x}^{\prime} .
\end{aligned}
$$

We have here adopted Einstein's convention of sums and $i, j \in\{x, y, z\}$ denotes the component of the vectors and tensors. Equations 7 and 8 together show how to model electromagnetic fields in a background model with known Green's tensors, due to an electric source. The Green's tensors depend upon the wavenumber $k=\sqrt{\mathrm{i} \omega \mu \tilde{\sigma}}$, which needs to be chosen with the appropriate sign to satisfy Sommerfeld's radiation condition (Colton and Kress, 1992; Zhdanov, 2002). 
Consider now a background model $\mathcal{B}$ with given parameters, $\sigma_{\mathbf{0}}, \epsilon_{0}, \mu_{0}$, as described above, and assume there in $\mathcal{B}$ is a domain $\mathcal{D}$ of finite volume which contains an inhomogeneity, or anomaly, compared to the electric conductivity, $\sigma$, of the background model. In our setting, the inhomogeneity in $\mathcal{D}$ might represent the hydrocarbon reservoir. Assume $\mathcal{D}$ is illuminated by an electric source from the outside, and on a domain $\mathcal{S}$ outside $\mathcal{D}$ receivers record the scattered field due to the conductivity contrast. In Figure 2 an intersection in the $x z$-plane of the model described is displayed. Now, from equations 7 and 8 we can calculate the incident electric and magnetic field in the domain $\mathcal{D}$ due to an electric source, $\mathbf{e}_{i}^{\mathrm{inc}}$ and $\mathbf{h}_{i}^{\mathrm{inc}}$, respectively.

We denote the conductivity inside $\mathcal{D}$ by $\sigma$. It is well known that in $\mathcal{D}$ the electric field satisfies (Abubakar and van den Berg, 2004; Gao, 2005)

$$
\mathbf{e}_{i}(\mathbf{x})=\mathbf{e}_{i}^{\mathrm{inc}}(\mathbf{x})+\int_{\mathcal{D}} \mathbf{G}_{i j}^{E}\left(\mathbf{x}, \mathbf{x}^{\prime}\right) \sigma_{0, v}\left(\mathbf{x}^{\prime}\right) \chi_{j j}\left(\mathbf{x}^{\prime}\right) \mathbf{e}_{j}\left(\mathbf{x}^{\prime}\right) \mathrm{d} \mathbf{x}^{\prime}, \quad \mathbf{x} \in \mathcal{D}
$$

which is the equivalent to the Lippmann-Schwinger equation for the scalar Helmholtz equation (Colton and Kress, 1992). Here $\mathbf{e}_{i}^{\text {inc }}(\mathbf{x})$ represents the incident electric field from the source, while the integral term represents the scattered field. The contrast $\chi$ is given by

$$
\chi=\left(\begin{array}{ccc}
\chi_{h} & 0 & 0 \\
0 & \chi_{h} & 0 \\
0 & 0 & \chi_{v}
\end{array}\right),
$$

where $\chi_{h}=\frac{\sigma_{h}}{\sigma_{0, v}}-\Upsilon$ and $\chi_{v}=\frac{\sigma_{v}}{\sigma_{0, v}}-1$. When the total electric field inside $\mathcal{D}$ is established we may calculate the scattered field at receiver locations by (Abubakar 
and van den Berg, 2004)

$$
\begin{aligned}
& \mathbf{f}_{i}^{E}(\mathbf{x})=\int_{\mathcal{D}} \mathbf{G}_{i j}^{E}\left(\mathbf{x}, \mathbf{x}^{\prime}\right) \sigma_{0, v}\left(\mathbf{x}^{\prime}\right) \chi_{j j}\left(\mathbf{x}^{\prime}\right) \mathbf{e}_{j}\left(\mathbf{x}^{\prime}\right) \mathrm{d} \mathbf{x}^{\prime}, \quad \\
& \mathbf{f}_{i}^{H}(\mathbf{x})=\mathcal{S}, \\
& =\int_{\mathcal{D}} \mathbf{G}_{i j}^{H}\left(\mathbf{x}, \mathbf{x}^{\prime}\right) \sigma_{0, v}\left(\mathbf{x}^{\prime}\right) \chi_{j j}\left(\mathbf{x}^{\prime}\right) \mathbf{e}_{j}\left(\mathbf{x}^{\prime}\right) \mathrm{d} \mathbf{x}^{\prime}, \quad \mathbf{x} \in \mathcal{S} .
\end{aligned}
$$

Thus, if we know the Green's tensors of the background medium we can model the scattered field from the inhomogeneity at a receiver location using equations 9-12.

For simplicity we introduce the vectors $\mathbf{e}=\left(\mathbf{e}_{x}, \mathbf{e}_{y}, \mathbf{e}_{z}\right)^{T}, \mathbf{f}^{E}=\left(\mathbf{f}_{x}^{E}, \mathbf{f}_{y}^{E}, \mathbf{f}_{z}^{E}\right)^{T}$ and $\mathbf{f}^{H}=\left(\mathbf{f}_{x}^{H}, \mathbf{f}_{y}^{H}, \mathbf{f}_{z}^{H}\right)^{T}$ and rewrite equations 9-12 in operator form

$$
\begin{aligned}
\mathbf{e} & =\mathbf{e}^{\mathrm{inc}}+G^{E, \mathcal{D}} \chi \mathbf{e}, \\
\mathbf{f}^{E} & =G^{E, \mathcal{S}} \chi \mathbf{e}, \\
\mathbf{f}^{H} & =G^{H, \mathcal{S}} \chi \mathbf{e},
\end{aligned}
$$

where now $G^{E, \mathcal{D}}, G^{E, \mathcal{S}}, G^{H, \mathcal{S}}$ are integral operators.

\section{Contrast Source Inversion}

We now wish to solve the inverse problem of estimating $\chi$ inside $\mathcal{D}$ given the scattered fields, that is to determine the conductivity profile of $\mathcal{D}$. We introduce the set of contrast sources, defined as (Abubakar et al., 2004b)

$$
\mathcal{W}=\left\{\mathbf{w}^{j, k}\right\}_{j=1 \ldots N_{s}}^{k=1 \ldots N_{f}}=\left\{\chi \mathbf{e}^{j, k}\right\}_{j=1 \ldots N_{s}}^{k=1 \ldots N_{f}}
$$

where $N_{s}$ is the total number of sources and $N_{f}$ is the number of frequencies. Intro- 
ducing this into equations 13-15 yields for a given source and frequency

$$
\begin{aligned}
\mathbf{e} & =\mathbf{e}^{\mathrm{inc}}+G^{E, \mathcal{D}} \mathbf{w}, \\
\mathbf{f}^{E} & =G^{E, \mathcal{S}} \mathbf{w}, \\
\mathbf{f}^{H} & =G^{H, \mathcal{S}} \mathbf{w} .
\end{aligned}
$$

We will from now use a norm similar to that associated with $L^{2}(\mathrm{~A})$, where $\mathrm{A}$ is some domain, which is given by

$$
\|f\|_{\mathrm{A}}^{2}=\int_{\mathrm{A}}|f(\mathbf{x})|^{2} \mathrm{~d} \mathbf{x}
$$

For more information on the norms, confer to Abubakar and van den Berg (2004).

The contrast source inversion (CSI) problem is formulated as a minimization problem as follows (Abubakar and van den Berg, 2004):

Find the contrast $\chi$, and the set of contrast sources $\mathcal{W}=\left\{\mathbf{w}^{j, k}\right\}_{j=1 \ldots N_{\mathrm{s}}}^{k=1 \ldots N_{\mathrm{f}}}$, such that the cost functional

$$
\begin{aligned}
F_{1}(\mathcal{W}, \chi) & =\alpha_{1}^{E} \sum_{k=1}^{N_{\mathrm{f}}} \sum_{j=1}^{N_{\mathrm{s}}}\left\|\Xi^{E, j, k}\left(\mathbf{f}^{E, j, k}-G^{E, \mathcal{S}, k} \mathbf{w}^{j, k}\right)\right\|_{\mathcal{S}}^{2} \\
& +\alpha_{1}^{H} \sum_{k=1}^{N_{\mathrm{f}}} \sum_{j=1}^{N_{\mathrm{s}}}\left\|\Xi^{H, j, k}\left(\mathbf{f}^{H, j, k}-G^{H, \mathcal{S}, k} \mathbf{w}^{j, k}\right)\right\|_{\mathcal{S}}^{2} \\
& +\alpha_{2} \sum_{k=1}^{N_{\mathrm{f}}} \sum_{j=1}^{N_{\mathrm{s}}}\left\|\chi \mathbf{e}^{\text {inc,j,k}}-\mathbf{w}^{j, k}+\chi G^{E, \mathcal{D}, k} \mathbf{w}^{j, k}\right\|_{\mathcal{D}}^{2}
\end{aligned}
$$

is minimized.

In this formulation, the two first terms encourage data fidelity, while the last term encourages the solution to solve equation 17 . This last term may be viewed as 
a physical regularization (Abubakar and van den Berg, 2004). Here $\alpha_{1}^{E}, \alpha_{1}^{H}, \alpha_{2}$ are normalization factors with reciprocals given by

$$
\begin{aligned}
\alpha_{1}^{E^{-1}} & =\sum_{k=1}^{N_{\mathrm{f}}} \sum_{j=1}^{N_{\mathrm{s}}}\left\|\Xi^{E, j, k} \mathbf{f}^{E, j, k}\right\|_{\mathcal{S}}^{2}, \\
\alpha_{1}^{H^{-1}} & =\sum_{k=1}^{N_{\mathrm{f}}} \sum_{j=1}^{N_{\mathrm{s}}}\left\|\Xi^{H, j, k} \mathbf{f}^{H, j, k}\right\|_{\mathcal{S}}^{2}, \\
\alpha_{2}^{-1} & =\sum_{k=1}^{N_{\mathrm{f}}} \sum_{j=1}^{N_{\mathrm{s}}}\left\|\chi \mathbf{e}^{\mathrm{inc}, j, k}\right\|_{\mathcal{D}}^{2},
\end{aligned}
$$

and $\Xi$ are data weights. The data weights may be chosen e.g. as window functions to remove unwanted noisy areas in the data or to emphasize some offset ranges. In our examples, the prior choice has been applied to small $(\leq 1000 \mathrm{~m})$ and large $(\geq 10000 \mathrm{~m})$ offsets, and at particularly noisy data. This is performed by assigning particularly low weights to these datapoints such that they have no influence on the inversion. The latter choice may be preferred in more complex cases where data from some offsets are considered more important than others.

To solve the CSI problem we follow Abubakar and van den Berg (2004); Abubakar et al. (2004b) and use the following iterative, alternating scheme, which approximates the full non linear problem by two linear problems: 
Input: Initial contrast and contrast sources

foreach iteration do

foreach frequency do

foreach source do

| Minimize equation 21 with respect to $\mathbf{w}^{j, k}$

\section{end}

end

Minimize equation 21 with respect to $\chi$

\section{end}

We will from now denote $\mathbf{w}^{(n), j, k}$ and $\chi^{(n)}$ as the estimates for the contrast sources and the contrast after iteration $n$. For the minimization with respect to the contrast sources, we follow Abubakar and van den Berg (2004) and use a conjugate gradient (CG) method as described by van den Berg (1984). In each iteration we do one CG step to the Euler-Lagrange equation for the contrast source $j, k$ :

$$
\begin{gathered}
{\left[\alpha_{1}^{E}\left(\Xi^{E, j, k} G^{E, \mathcal{S}, k}\right)^{*}\left(\Xi^{E, j, k} G^{E, \mathcal{S}, k}\right)+\alpha_{1}^{H}\left(\Xi^{H, j, k} G^{H, \mathcal{S}, k}\right)^{*}\left(\Xi^{H, j, k} G^{H, \mathcal{S}, k}\right)\right.} \\
\left.+\alpha_{2} R^{(n), *} R^{(n)}\right] \mathbf{w}^{(n), j, k}= \\
\alpha_{1}^{E}\left(\Xi^{E, j, k} G^{E, \mathcal{S}, k}\right)^{*} \Xi^{E, j, k} \mathbf{f}^{E, j, k}+\alpha_{1}^{H}\left(\Xi^{H, j, k} G^{H, \mathcal{S}, k}\right)^{*} \Xi^{H, j, k} \mathbf{f}^{H, j, k} \\
+\alpha_{2} R^{(n), *} \chi^{(n-1)} \mathbf{e}^{\mathrm{inc}, j, k},
\end{gathered}
$$

where $R^{(n)}=I-\chi^{(n-1)} G^{E, \mathcal{D}, k}$ and the superscript $*$ denotes the Hilbert adjoint operator. 
The minimization with respect to $\chi$ may be carried out explicitly, and we find

$$
\begin{aligned}
{\left[\sum_{k=1}^{N_{\mathrm{f}}} \sum_{j=1}^{N_{\mathrm{s}}}\left|\mathbf{e}_{x}^{(n), j, k}\right|^{2}+\left|\mathbf{e}_{y}^{(n), j, k}\right|^{2}\right] \chi_{h}^{(n)} } & =\sum_{k=1}^{N_{\mathrm{f}}} \sum_{j=1}^{N_{\mathrm{s}}} \overline{\mathbf{e}_{x}^{(n), j, k}} \mathbf{w}_{x}^{(n), j, k}+\overline{\mathbf{e}_{y}^{(n), j, k}} \mathbf{w}_{y}^{(n), j, k},(26) \\
{\left[\sum_{k=1}^{N_{\mathrm{f}}} \sum_{j=1}^{N_{\mathrm{s}}}\left|\mathbf{e}_{z}^{(n), j, k}\right|^{2}\right] \chi_{v}^{(n)} } & =\sum_{k=1}^{N_{\mathrm{f}}} \sum_{j=1}^{N_{\mathrm{s}}} \overline{\mathbf{e}_{z}^{(n), j, k}} \mathbf{w}_{z}^{(n), j, k}
\end{aligned}
$$

where $\mathbf{e}_{x}^{(n), j, k}$ is the $x$-component of the vector $\mathbf{e}^{\mathrm{inc}, j, k}+G^{E, \mathcal{D}, k} \mathbf{W}^{(n), j, k}$, and the overline denotes the complex conjugate.

We note that the constant $\alpha_{2}$ needs to be updated in each iteration. In these calculations we have for simplicity in iteration $n$ let

$$
\alpha_{2}^{(n)^{-1}}=\sum_{k=1}^{N_{\mathrm{f}}} \sum_{j=1}^{N_{\mathrm{s}}}\left\|\chi^{(n-1)} \mathbf{e}^{\mathrm{inc}, j, k}\right\|_{\mathcal{D}}^{2}
$$

such that it is a constant in each iteration.

\section{RESULTS}

The electromagnetic inverse problem is a notoriously ill-posed problem (Zhdanov, 2002; Feng et al., 2003; Abubakar et al., 2004b) in the Hadamard sense (McOwen, 1996). Due to this we introduce a regularizing term linearly into the cost functional to be able to incorporate prior information on the contrast. We now assume that $\mathcal{D}$ is discretized into $N_{p}$ cells, arrange $\chi$ into a vector of size $3 \times N_{p}, \chi=\left(\chi_{h}, \chi_{h}, \chi_{v}\right)^{T}$, and introduce a new optimization problem as

$$
\underset{\mathcal{W}, \chi}{\operatorname{argmin}}\left(F_{1}(\mathcal{W}, \chi)+\lambda^{2} F_{2}(\chi)\right)=\underset{\mathcal{W}, \chi}{\operatorname{argmin}}\left(F_{1}(\mathcal{W}, \chi)+\lambda^{2}\left\|\Omega\left(\chi-\chi^{\text {ref }}\right)\right\|_{\mathcal{D}}^{2}\right) .
$$


Here $F_{2}$ is introduced to be able to incorporate prior information concerning the model. Further, $\chi^{\text {ref }}$ is some reference model, e.g. expected solution, $\Omega$ is some weighting matrix and $\lambda \in \mathbb{R}$ a weighting parameter controlling the tradeoff between $F_{1}(\mathcal{W}, \chi)$ and $F_{2}(\chi)$ which needs to be chosen appropriately not to emphasize only one of the terms.

The weights $\Omega$ are given by the matrix

$$
\Omega=\left(\begin{array}{lll}
\Omega_{11} & \Omega_{12} & \Omega_{13} \\
\Omega_{21} & \Omega_{22} & \Omega_{23} \\
\Omega_{31} & \Omega_{32} & \Omega_{33}
\end{array}\right),
$$

where $\Omega_{i j}$ are $N_{p} \times N_{p}$ sub-matrices. They can be chosen to introduce different effects, e.g.

- Freeze parts of the model by choosing the diagonal elements of $\Omega_{i i}$ large and $\Omega$ elsewise zero, thus keeping the reconstruction close to the reference model.

- Introduce spatial correlations between cells by choosing off-diagonal cells nonzero in some of the sub-matrices.

- Introduce structural information, allowing for sharp boundaries where interfaces are believed to occur, by applying smoothing filters to gridcells away from the interface.

- Introduce correlations between $\chi_{h}$ and $\chi_{v}$ by choosing $\Omega_{13}, \Omega_{23}, \Omega_{31}, \Omega_{32}$ nonzero. 
In our examples $\Omega$ is chosen to be second order smoothing filters in the horizontal and vertical directions acting on $\chi_{h}$ and $\chi_{v}$ individually, thus introducing spatial correlations between neighboring cells. Further, the reference model is $\chi^{\text {ref }}=0$ to emphasize only the smoothness of the solution. Different weights are not put on horizontal and vertical smoothing as the purpose here is numerical stabilization. The parameter $\lambda$ is chosen by observation such that the term $F_{2}$ does not dominate the cost functional, and it is relaxed towards zero as the iterations proceed to not over emphasize the prior-term. Other strategies for choosing $\lambda$ are mentioned in the discussion.

We note that this second term does not affect the estimation of $\mathbf{w}^{j, k}$. However, the estimators for $\chi_{h}$ and $\chi_{v}$ are changed, and due to the spatial correlations equations 26 and 27 no longer hold point wise. Thus, in each iteration we must solve a linear system of equations specified by the correlations determined by $\Omega$ to estimate the contrast.

We now demonstrate the method on two datasets from the Troll field on the Norwegian North Sea. Since both surveys were collected along lines the results are displayed as slices along the source/receiver line.

\section{Troll 2003}

The first dataset was collected by Statoil over the Troll West Gas Province (TWGP) in 2003, and the setting is described in Johansen et al. (2005). The source was a dipole of 
$230 \mathrm{~m}$ length and a peak current of approximately 1000A. The fundamental frequency was $0.25 \mathrm{~Hz}$, and although several harmonics were recorded only the fundamental frequency is used in this inversion, as using higher frequencies did not make large differences in the image of TWGP. 24 receivers were dropped on the seabed, all of which measured the inline electric field. A subset of the receivers also measured the crossline magnetic field.

The background model consists of a sea layer, with depth equal to the arithmetic mean of the receiver depths and conductivity $3.3 \mathrm{~S} / \mathrm{m}$, and a homogeneous halfspace with conductivity $0.35 \mathrm{~S} / \mathrm{m}$. The halfspace conductivity was chosen by modeling comparison with the real data. Upon inspecting the seismic section the domain $\mathcal{D}$ was chosen as $-6000 \mathrm{~m} \leq x \leq 7750 \mathrm{~m},-1000 \mathrm{~m} \leq y \leq 1000 \mathrm{~m}$ and $1000 \mathrm{~m} \leq z \leq 2000 \mathrm{~m}$ with grid size $\Delta x=250 \mathrm{~m}, \Delta y=250 \mathrm{~m}, \Delta z=50 \mathrm{~m}$. The initial model was chosen as shown in Figure 3, which is seen to be shorter and more shallow than the reservoir.

The result from the isotropic inversion is displayed in Figure 4 laid over a seismic section. We observe that the anomaly is moved slightly deeper and extended laterally compared to the initial model, and that it corresponds well with the seismic image. For this case we also present the results when the domain $\mathcal{D}$ is extended $2 \mathrm{~km}$ in each direction laterally and $500 \mathrm{~m}$ in each direction vertically compared to the domain described above. This is shown in Figure 5. We see that the results are very similar, which might indicate that the background model is well chosen.

The results from the TIV inversion are displayed in Figure 6. We observe that also the TIV inversion move the anomaly slightly deeper and extends it laterally 
to approximately the same position as the isotropic inversion. From Figure 6(a) and Figure 6(b) we observe that almost no contrast is estimated for $\sigma_{h}$. This is as expected since the signal propagation in the reservoir is governed by the vertical conductivity $\sigma_{v}$ (Løseth, 2007), and thus when we apply a smoothing filter the profile for $\sigma_{h}$ will be smoothed towards zero. The contrast estimated for the vertical conductivity is larger than that estimated in the isotropic case.

\section{Troll 2008}

The second dataset was collected over the Troll field in 2008 at a slightly different location than the example above. The survey was at the same location and similar to the survey described in Farrelly et al. (2004); Johnstad et al. (2005). This line now crosses three laterally separated anomalies as shown in Figure 2 in Farrelly et al. (2004), the largest being the TWGP, but also the Troll West Oil Province (TWOP) further west which lies slightly deeper and has less contrast. The inversion was now carried out on the fundamental frequency and it's first odd harmonic, i.e. $0.25 \mathrm{~Hz}$ and $0.75 \mathrm{~Hz}$, as the fundamental frequency alone was not able to pick up the smaller anomalies. Further, the smaller anomalies only appeared after a sufficient level of data misfit was achieved. All receivers measured the inline electric and crossline magnetic fields.

The background model again consists of a sea layer, with depth equal to the arithmetic mean of the receiver depths and conductivity $3.3 \mathrm{~S} / \mathrm{m}$, and a homogeneous halfspace with conductivity $0.35 \mathrm{~S} / \mathrm{m}$. Upon inspecting the seismic section the domain 
$\mathcal{D}$ was chosen as $-10000 \mathrm{~m} \leq x \leq 12000 \mathrm{~m},-1000 \mathrm{~m} \leq y \leq 1000 \mathrm{~m}$ and $1000 \mathrm{~m} \leq z \leq$ $2000 \mathrm{~m}$ with grid size $\Delta x=250 \mathrm{~m}, \Delta y=250 \mathrm{~m}, \Delta z=50 \mathrm{~m}$. The initial model was chosen as a rectangular, resistive body placed between $7000 \mathrm{~m} \leq x \leq 8750 \mathrm{~m}, 1600 \mathrm{~m} \leq$ $z \leq 1650 \mathrm{~m}$ as shown in Figure 7. A small anomaly is expected approximately at this position as shown in Figure 2 in Farrelly et al. (2004).

The results are displayed in Figures 8 and 9, which show the isotropic and TIV inversion results, respectively. The same effect as in the previous example concerning the estimate for $\sigma_{h}$ is observed in Figure 9(a). Figure 8 shows that the initial box only remains with a weak contrast, while the large anomaly connected to TWGP is introduced. However, Figure 9(b) displays a brighter anomaly in $\sigma_{v}$ at the approximate position of the initial model, the large anomaly from TWGP is introduced, and even a small anomaly to the left of TWGP, approximately at the position of the TWOP. The large anomaly from the TIV inversion is however more spread out than the isotropic estimate.

We again observe the nice fit with the seismic. However, the small anomaly close to TWOP in Figure 9(b) is slightly too close to TWGP. This might be an effect due to the bias introduced by the smoothing regularization, but could also lead to questioning this anomaly. The slightly red color in Figure 9(a) might indicate that the background model is not optimal, and illustrates the difficulty of choosing an appropriate background model. This will be further mentioned in the discussion.

The additional degrees of freedom introduced by allowing a TIV in stead of isotropic medium naturally results in a better data fit. The term in the cost functional 
corresponding to fit in e decrease from 0.0384 to 0.0341 , and the term corresponding to $\mathbf{h}$ from 0.0234 to 0.0226 . This may also be a part of the explaination for why the smaller anomalies appear in the TIV inversion, but not in the isotropic.

\section{DISCUSSION}

Although we have found the CSI method to be applicable to real field data we may point at several critical points with this method that may lead to difficulties in the inversion. The most critical point is choosing an appropriate background model, which in turn means having a good perception of the over- and underburden. If it is not appropriately chosen the algorithm will attempt to explain these effects within the domain $\mathcal{D}$, and thus lead to an incorrect inversion. This is especially important if the anomalies are small or have weak contrasts. The theory presented is not limited to a planarly layered background, as is chosen in the examples. However, with this choice the Green's tensors and incident fields may be calculated very rapidly, ensuring a fast algorithm under these limitations.

Ideally one would like to invert for the whole subsurface simultaneously, but this will normally give a variety of resulting models with different background resitivities in combination with different structures of anomalies, depending on different prior data interpretations. Thus, one finally has to select the most probable models based on seismics and possibly well logs. CSI is a suitable tool for investigating if the mCSEM data contain responses from an anomaly for a suite of probable background models, and may thus be helpful in this final selection. 
When introducing prior information on the model several issues needs to be addressed. Obviously an appropriate weighting matrix $\Omega$ needs to be chosen. In our examples we used this term only for numerical stabilization, and thus chose equal weights on horizontal and vertical smoothing and $\lambda$ such that the prior term did not dominate the cost functional. However, it may be argued that different weights should be put on horizontal and vertical smoothing due to layering in the earth. Further, if the prior information should be appropriately emphasized $\lambda$ needs to be carefully chosen, and several strategies have been investigated for this. A particular choice is to use the L-curve criterion (Hansen and O'leary, 1993), which involves solving the inverse problem for several different $\lambda$ and is thus very time consuming for large-scale geophysical inverse problems. A fast approach to approximating this value was developed by Rabben and Ursin (2007), cast in a Bayesian setting. Zhdanov (2002) suggests choosing the regularization parameter according to an estimated noise level in the weighted data, and also cross validation techniques have been studied, see e.g. Tenorio (2001) for a review and the references therein. All these choices are valid and demonstrates the difficulties of choosing an appropriate parameter.

Finally, we wish to emphasize the importance of analyzing the data and prior information before performing an inversion. Determining which parameters can be imaged and being aware of the bias introduced through the chosen regularization is important, and should be taken into account also when analyzing the output from inversion algorithms. Several inversions using different regularizations and different parameters should be performed to study the consistency of the results. 


\section{CONCLUSIONS}

We have demonstrated a CSI inversion method for mCSEM experiments in TIV anisotropic media. The method is tested in two real field examples, both from the Troll field offshore Norway.

The method is based on an integral equation framework, thus relying on choosing an appropriate background model. It is formulated as an optimization problem minimizing a cost functional which enforces both data fidelity and that the solution fits with the electromagnetic equivalent of the Lippmann-Schwinger equation for the scalar Helmholtz equation. The latter term may be viewed as a natural regularizer.

A method for introducing prior information on the model is also introduced linearly into the cost functional. This may be used for e.g. numerical stabilization, structural information, keeping the solution close to an expected solution or correlating the horizontal and vertical conductivities. This regularization relies on choosing a regularization parameter, for which several strategies are discussed.

We found that the method is applicable to real field data, and the second example demonstrates that the TIV inversion is superior to the isotropic in some situations to properly image the subsurface. However, information concerning the background model is needed for a successful result. The method should thus be viewed in context with other methods which discretize the entire survey area. 


\section{ACKNOWLEDGEMENTS}

The authors wish to thank the Troll license for permission to publish this work. Torgeir Wiik acknowledges Statoil ASA for sponsoring his Ph.D. project. Bjørn Ursin has received financial support from VISTA and from the Norwegian Research Council through the ROSE project. 


\section{REFERENCES}

Abubakar, A., T. M. Habashy, V. L. Druskin, L. Knizhnerman, and D. Alumbaugh, 2008a, 2.5D forward and inverse modeling for interpreting low-frequency electromagnetic measurements: Geophysics, 73, F165-F177.

Abubakar, A., W. Hu, P. M. van den Berg, and T. M. Habashy, 2008b, A finitedifference contrast source inversion method: Inverse Problems, 24.

Abubakar, A., and P. M. van den Berg, 2002, The Contrast Source Inversion method for location and shape reconstructions: Inverse Problems, 18, 495-510.

— 2004 , Iterative forward and inverse algorithms based on domain integral equations for three-dimensional electric and magnetic objects: Journal of Computational Physics, 195, 236-262.

Abubakar, A., P. M. van den Berg, and J. J. Mallorqui, 2004a, Imaging of biomedical data using a multiplicative regularized Contrast Source Inversion method: IEEE Transactions on Microwave Theory and Techniques, 50, 1761-1771.

Abubakar, A., P. M. van den Berg, and S. Y. Semenov, 2004b, A robust interative method for Born inversion: IEEE Transactions on Geoscience and Remote Sensing, $42,342-354$.

Colton, D., and R. Kress, 1992, Inverse acoustic and electromagnetic inverse scattering theory: Springer Verlag.

Eidesmo, T., S. Ellingsrud, L. M. MacGregor, S. Constable, M. C. Sinha, S. Johansen, F. N. Kong, and H. Westerdal, 2002, Sea Bed Logging (SBL), a new method for remote and direct idetification of hydrocarbon filled layers in deepwater areas: First 
Break, 20, 144-152.

Farrelly, B., C. Ringstad, S. E. Johnstad, and S. Ellingsrud, 2004, Remote characterization of hyrdocarbon filled reservoirs at the Troll field by sea bed logging: EAGE Fall Research Workshop on Advances in Seismic Acquisition Technology.

Feng, H., V. Galdi, and D. A. Castañon, 2003, An object-based contrast source inversion method for homogeneous targets: Subsurface sensing technologies and applications, 4, 355-374.

Gao, G., 2005, Simulation of borehole electromagnetic measurements in dipping and anisotropic rock formations and inversion of array induction data: $\mathrm{PhD}$ thesis, University of Texas at Austin.

Green, G., 1828, An essay on the application of mathematical analysis to the theories of electricity and magnetism: T. Wheelhouse, Nottingham.

Hansen, P. C., and D. P. O'leary, 1993, The use of the L-curve in the regularization of discrete ill-posed problems: SIAM Journal on Scientific Computing, 14, 1487-1503.

Johansen, S. E., H. E. F. Amundsen, T. Røsten, S. Ellingsrud, T. Eidesmo, and A. H. Bhuyian, 2005, Subsurface hydrocarbons detected by electromagnetic sounding: First Break, 23, 31-36.

Johnstad, S. E., B. A. Farrely, and C. Ringstad, 2005, Em seabed logging on the troll field: EAGE 67th Conference \& Exhibition - Madrid, Spain.

Kong, F. N., S. E. Johnstad, T. Røsten, and H. Westerdal, 2008, A 2.5D finite-element-modeling difference method for marine csem modeling in stratified anisotropic media: Geophysics, 73, F9-F19.

Løseth, L. O., 2007, Modelling of controlled source electromagnetic data: PhD thesis, 
Norwegian University of Science and technology.

Løseth, L. O., and B. Ursin, 2007, Electromagnetic fields in planarly layered anisotropic media: Geophysical Journal International, 170, 44-80.

McOwen, R., 1996, Partial differential equations. methods and applications: PrenticeHall.

Newman, G., 1995, Crosswell electromagnetic inversion using integral and differential equations: Geophysics, 60, 899-911.

Newman, G. A., and G. M. Hoversten, 2000, Solution strategies for two and threedimensional electromagnetic inverse problems: Inverse Problems, 16, 1357-1375.

Rabben, T. E., and B. Ursin, 2007, Nonlinear least squares inversion of reflection coefficients using bayesian regularization: Presented at the Proceedings of the SEG Annual Meeting 2007.

Stratton, J. A., 1941, Electromagnetic theory: McGraw-Hill.

Tenorio, L., 2001, Statistical regularization of inverse problems: SIAM Review, 43, $347-366$.

Tikhonov, A. N., and V. Y. Arsenin, 1977, Solution of ill-posed problems: W.H.Winston and Sons.

Ursin, B., 1983, Review of elastic and electromagnetic wave propagation in horizontally layered media: Geophysics, 48, 1063-1081.

van den Berg, P. M., 1984, Iterative computational techniques in scattering based upon the integrated square error criterion: IEEE Transactions on Antennas and Propagation, 32, 1063-1071.

van den Berg, P. M., and R. E. Kleinman, 1997, A Contrast Source Inversion method: 
Inverse Problems, 13, 1607-1620.

Wannamaker, P. E., G. W. Hohmann, and W. A. SanFilipo, 1984, Electromagnetic modelling of three-dimensional bodies in layered earths using integral equations: Geophysics, 49, 60-74.

Ward, S. H., and G. W. Hohmann, 1987, Electromagnetic theory for geophysical applications, in Electromagnetic methods in applied geophysics: Society of Exploration Geophysicists, 131-311.

Zhdanov, M. S., 2002, Geophysical inverse theory and regularization problems: Elsevier. 
Figure 1: Figure displaying a usual configuration of a mCSEM survey. A horizontal electric dipole is towed behind a vessel, while receivers are placed on the seabed. Main sources for measured response are: 1:Reflection from sea-air interface, 2:direct wave, 3:Response from the seabed, 4:Response from hydrocarbon reservoir. The figure is retrieved from Løseth (2007).

Figure 2: Schematic intersection of the model.

Figure 3: Initial model for the 2003 Troll dataset. Triangles denote receiver positions, red rectangle marks main reservoir.

Figure 4: Isotropic inversion result for the 2003 Troll dataset laid over a seismic section. Triangles denote lateral receiver positions, red rectangle marks main reservoir. Figure 5: Isotropic results for the 2003 Troll dataset with different size on $\mathcal{D}$. Triangles denote lateral receiver positions. (a) Small $\mathcal{D}$, (b) Large $\mathcal{D}$.

Figure 6: TIV inversion results for the 2003 Troll dataset laid over a seismic section. Triangles denote lateral receiver positions, red rectangle marks main reservoir. (a) $\sigma_{h},(\mathrm{~b}) \sigma_{v}$.

Figure 7: Initial model for the 2008 Troll dataset laid over a seismic section. Triangles denote lateral receiver positions, red rectangles mark reservoirs.

Figure 8: Isotropic inversion result for the 2008 Troll dataset laid over a seismic section. Triangles denote lateral receiver positions, red rectangles mark reservoirs.

Figure 9: TIV inversion results for the 2008 Troll dataset laid over a seismic section. Triangles denote lateral receiver positions, red rectangles mark reservoirs. (a) $\sigma_{h}$, (b) $\sigma_{v}$. 


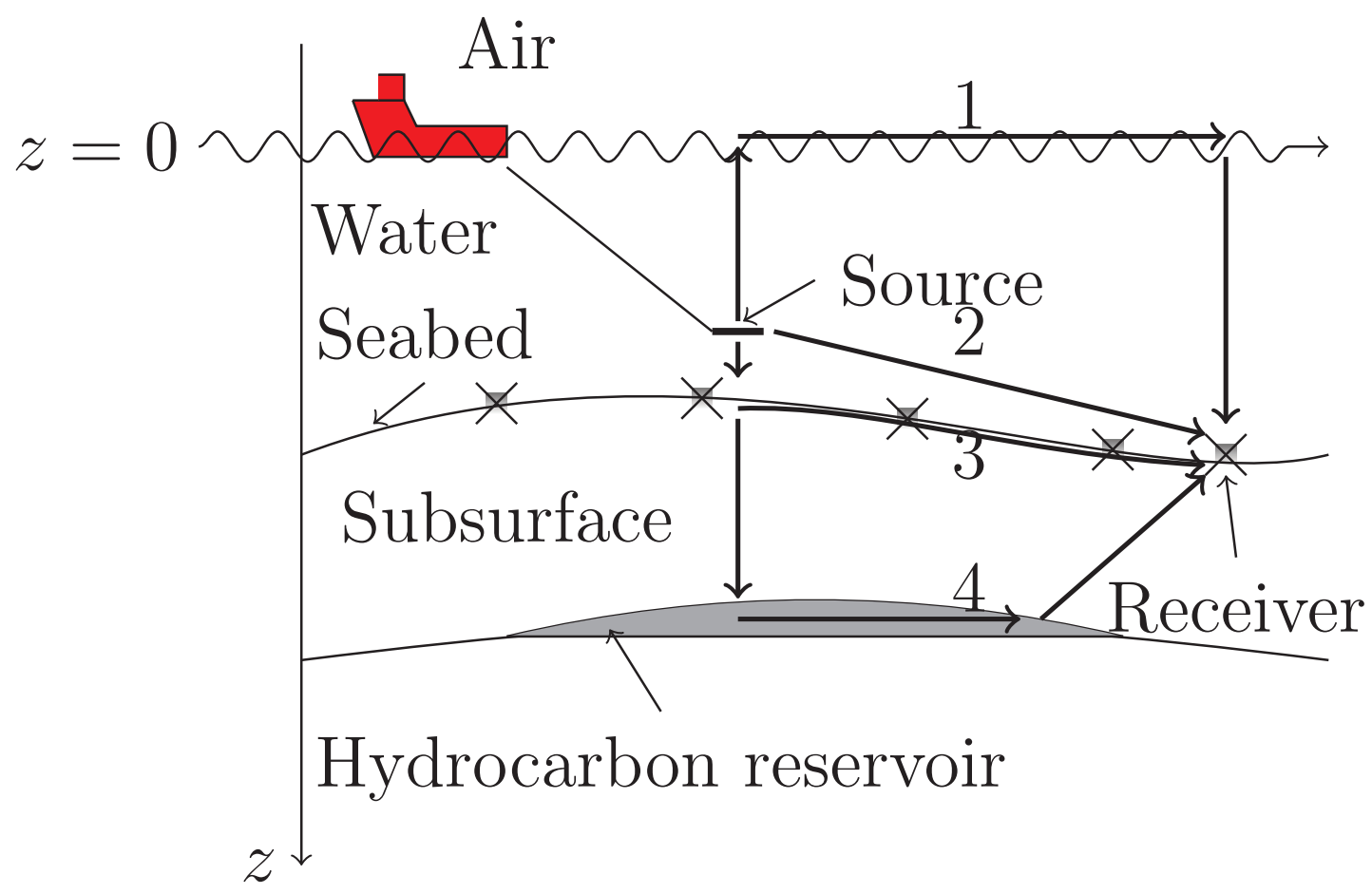

Figure 1: Figure displaying a usual configuration of a mCSEM survey. A horizontal electric dipole is towed behind a vessel, while receivers are placed on the seabed. Main sources for measured response are: 1:Reflection from sea-air interface, 2:direct wave, 3:Response from the seabed, 4:Response from hydrocarbon reservoir. The figure is retrieved from Løseth (2007). 


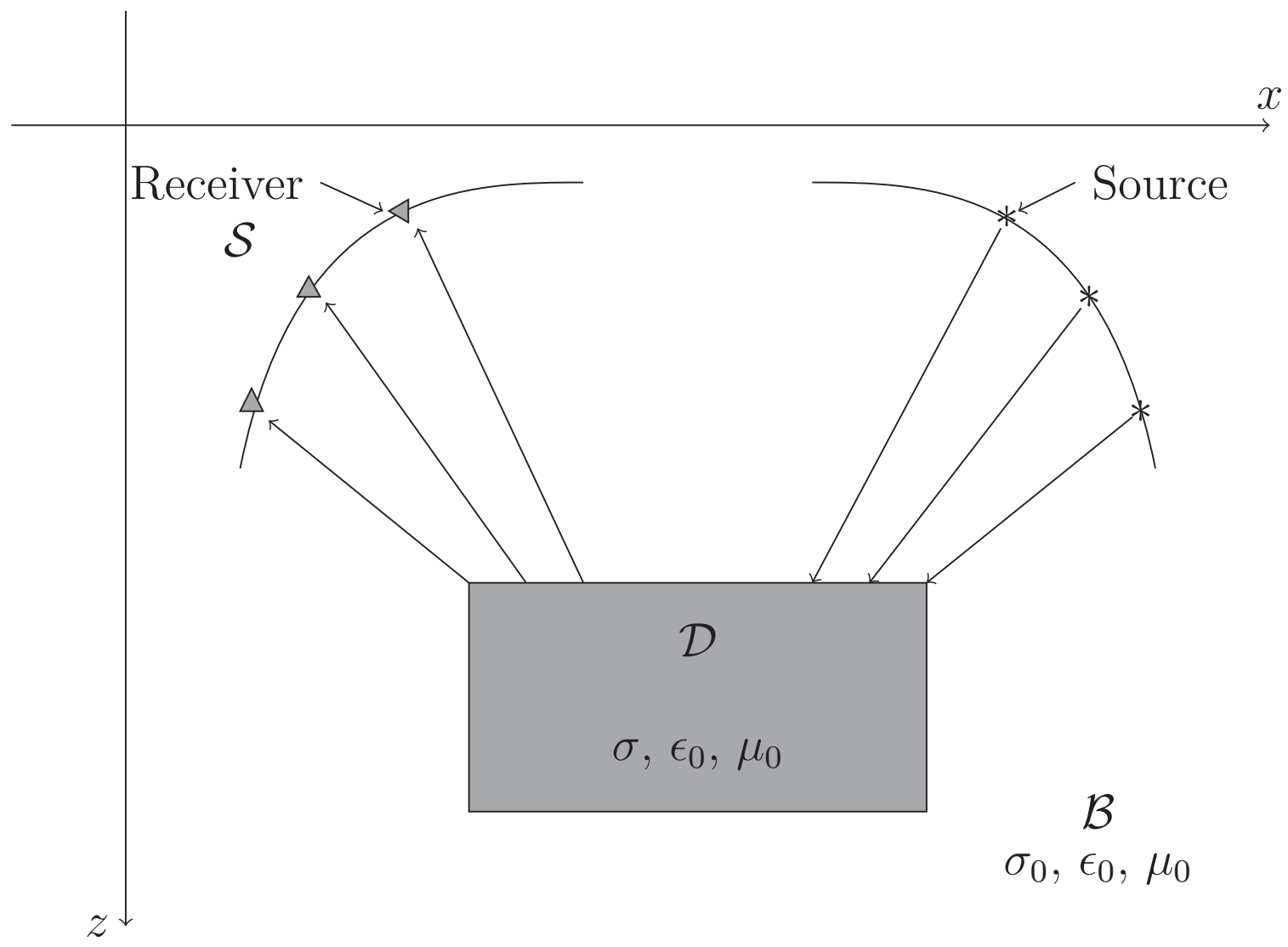

Figure 2: Schematic intersection of the model. 


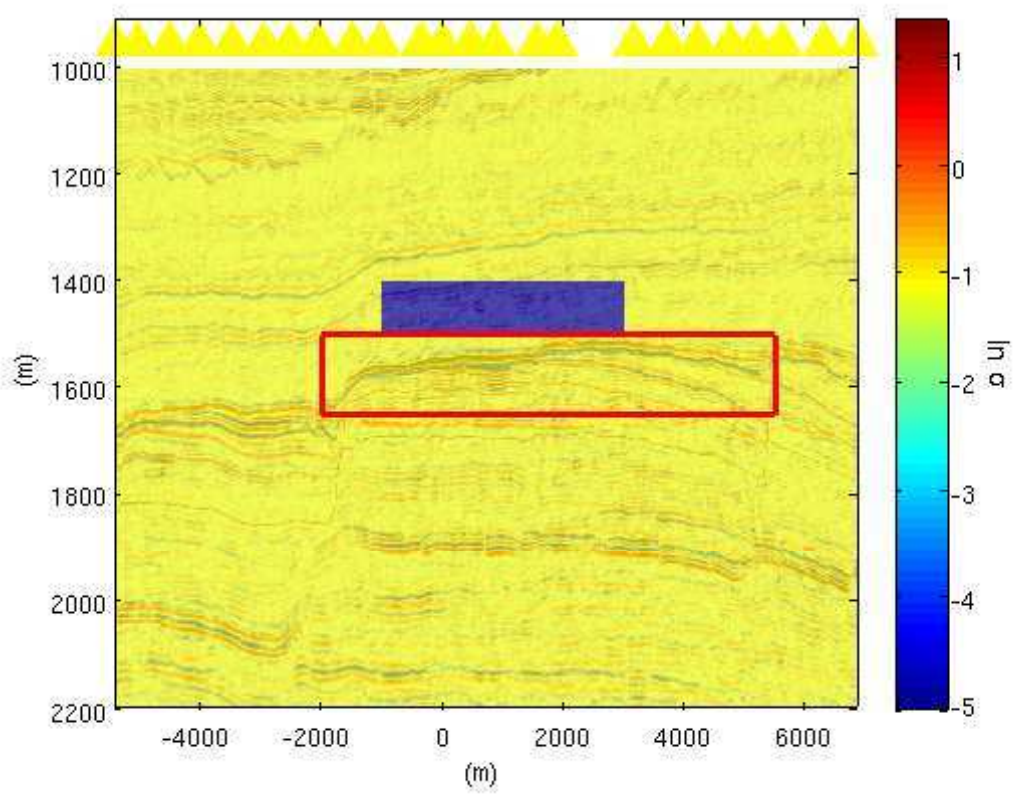

Figure 3: Initial model for the 2003 Troll dataset. Triangles denote receiver positions, red rectangle marks main reservoir. 


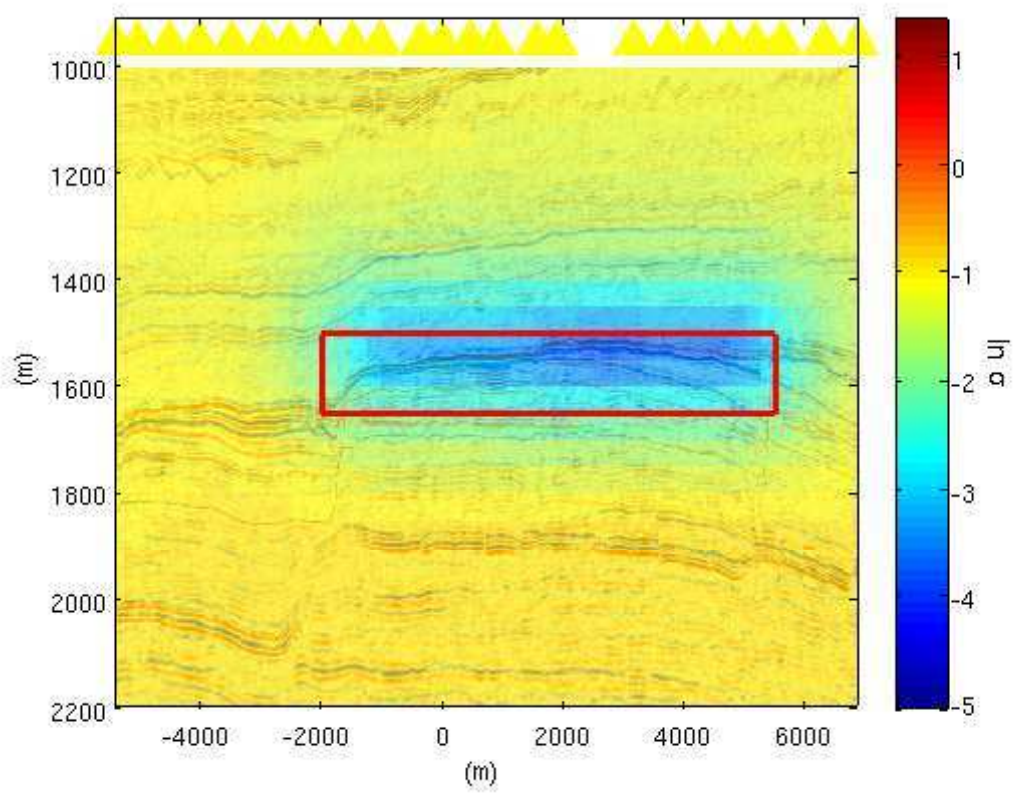

Figure 4: Isotropic inversion result for the 2003 Troll dataset laid over a seismic section. Triangles denote lateral receiver positions, red rectangle marks main reservoir. 
(a)

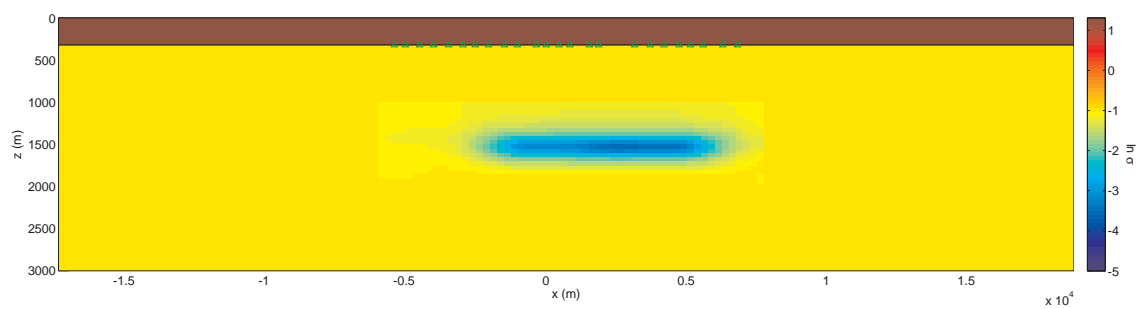

(b)

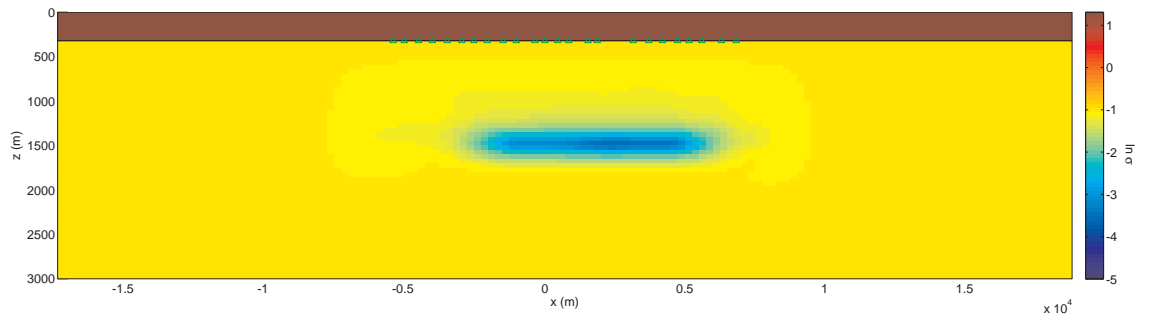

Figure 5: Isotropic results for the 2003 Troll dataset with different size on $\mathcal{D}$. Triangles denote lateral receiver positions. (a) $\operatorname{Small} \mathcal{D}$, (b) Large $\mathcal{D}$. 
(a)

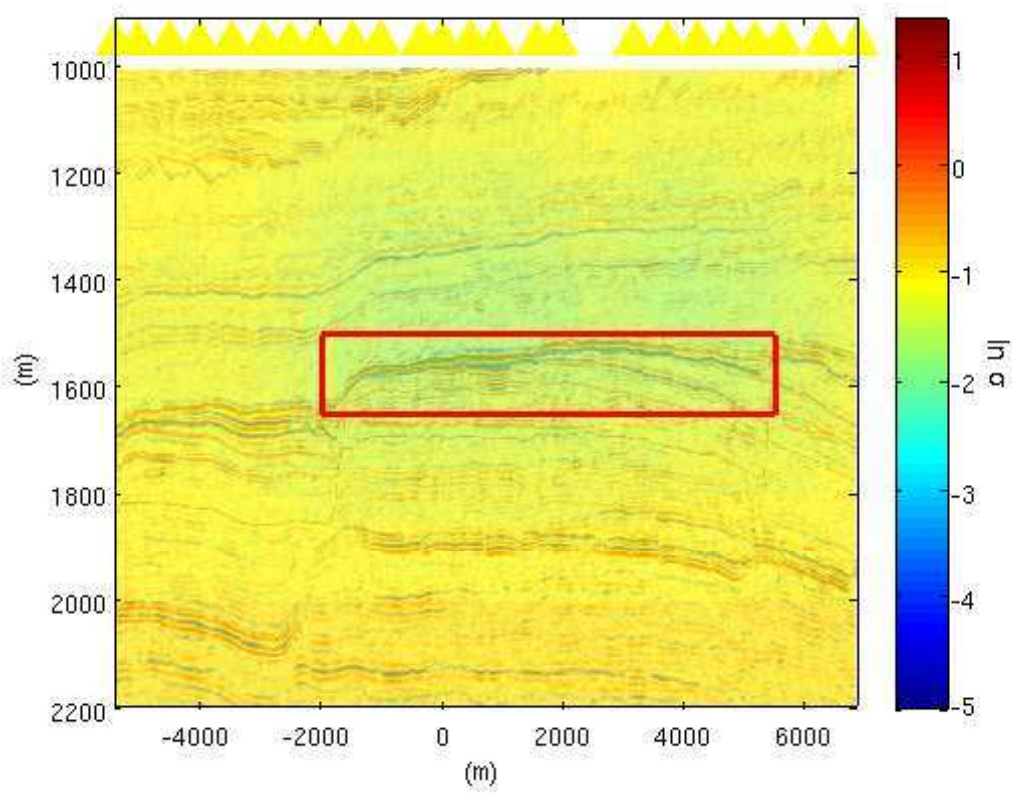

(b)

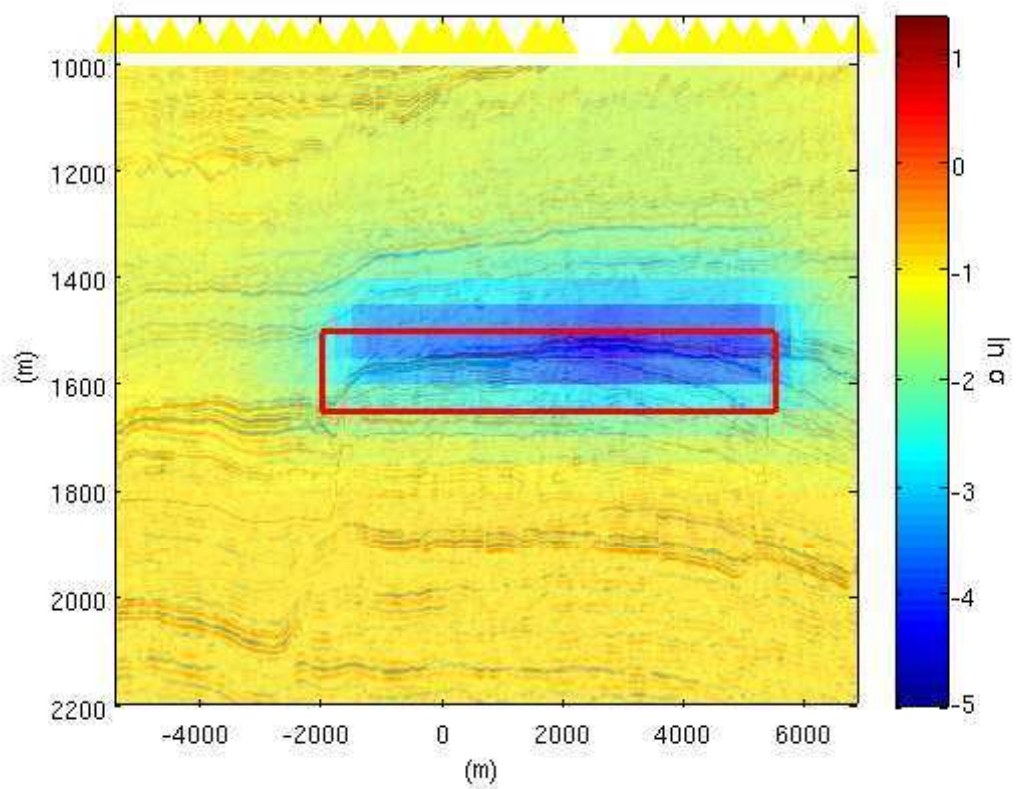

Figure 6: TIV inversion results for the 2003 Troll dataset laid over a seismic section.

Triangles denote lateral receiver positions, red rectangle marks main reservoir. (a) $\sigma_{h},(\mathrm{~b}) \sigma_{v}$. 


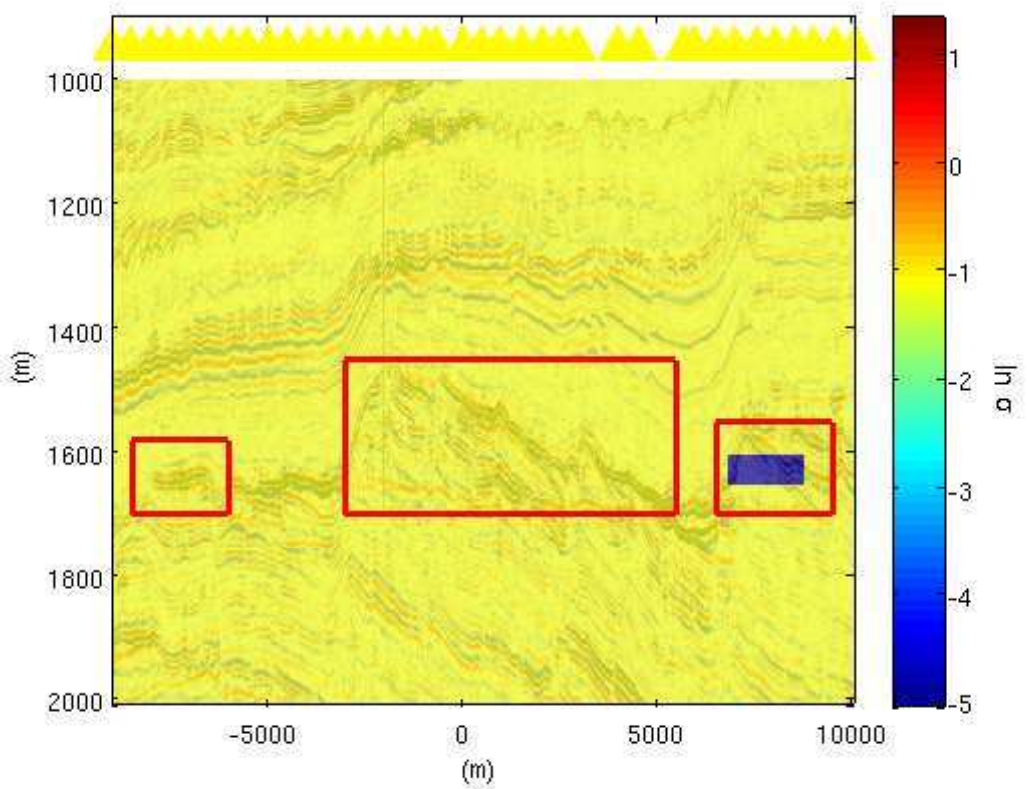

Figure 7: Initial model for the 2008 Troll dataset laid over a seismic section. Triangles denote lateral receiver positions, red rectangles mark reservoirs. 


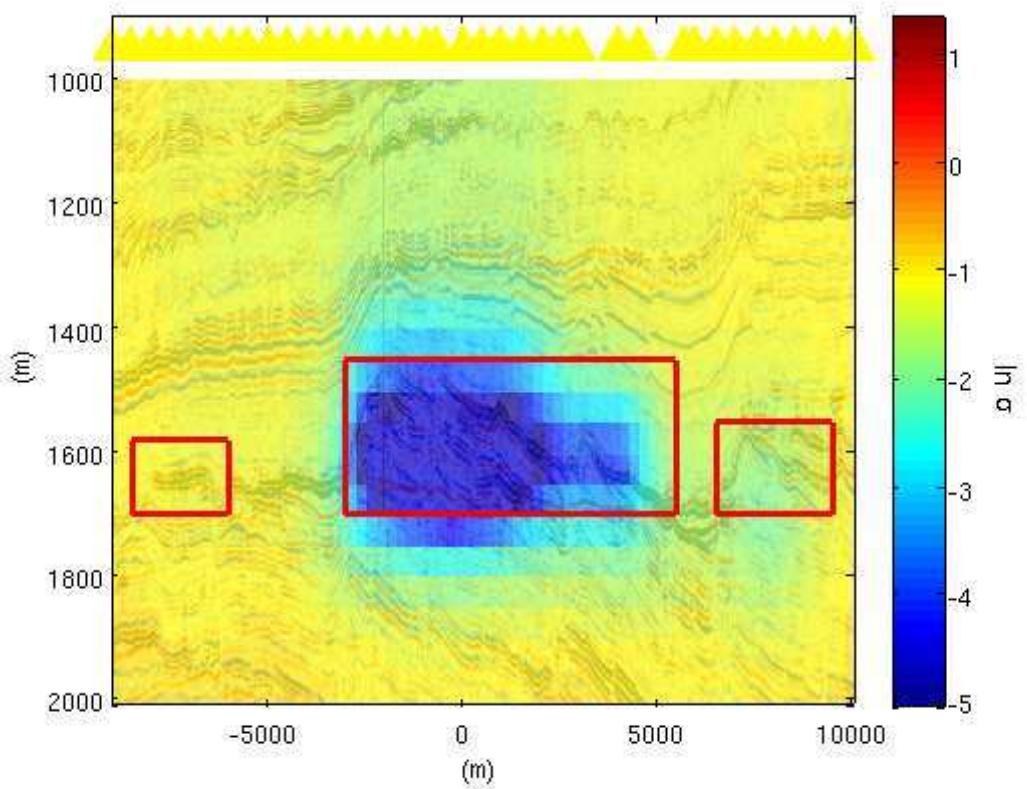

Figure 8: Isotropic inversion result for the 2008 Troll dataset laid over a seismic section. Triangles denote lateral receiver positions, red rectangles mark reservoirs. 
(a)

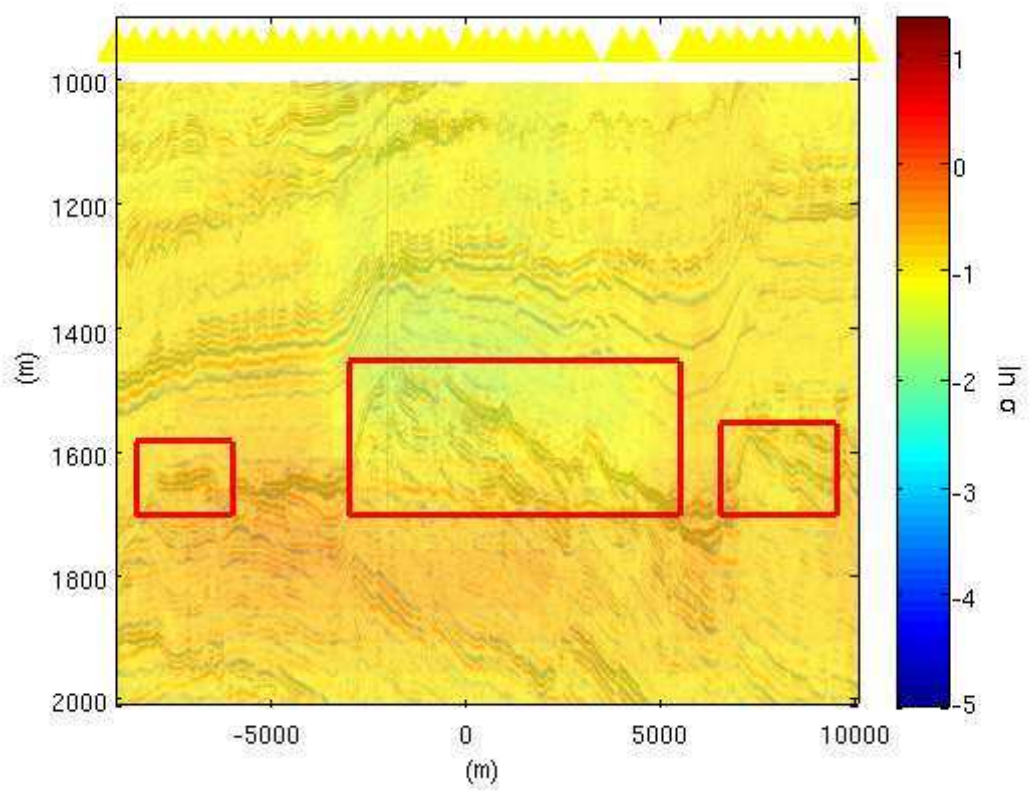

(b)

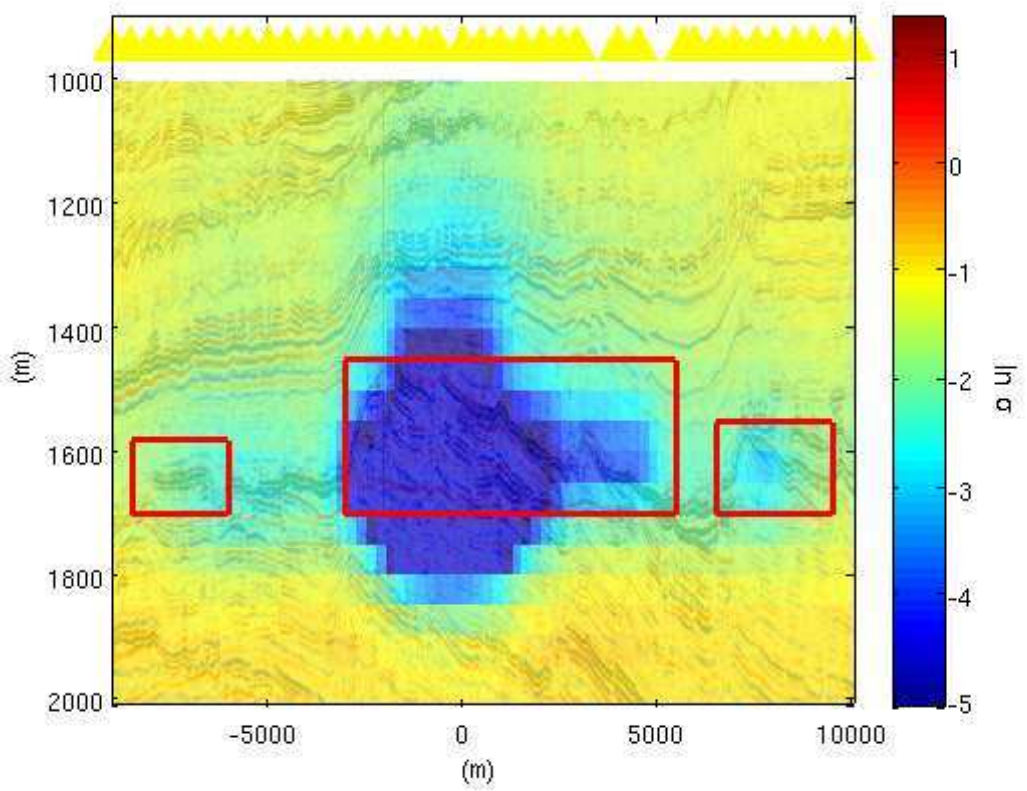

Figure 9: TIV inversion results for the 2008 Troll dataset laid over a seismic section.

Triangles denote lateral receiver positions, red rectangles mark reservoirs. (a) $\sigma_{h}$, (b) $\sigma_{v}$. 